Original Research Paper

\title{
Modeling the Error Term by Moving Average and Generalized Autoregressive Conditional Heteroscedasticity Processes
}

\author{
${ }^{1,2}$ Ayodele Abraham Agboluaje, ${ }^{1}$ Suzilah bt Ismail and ${ }^{3}$ Chee Yin Yip \\ ${ }^{I}$ School of Quantitative Sciences, College of Arts and Sciences, Universiti Utara Malaysia, Malaysia \\ ${ }^{2}$ Department of Mathematics and Computer Science, Faculty of Natural Sciences, \\ Ibrahim Badamasi Babangida University, Lapai, Nigeria \\ ${ }^{3}$ Department of Economics, Faculty of Business and Finance, Universiti Tuanku Abdul Rahman, Malaysia
}

Article history

Received: 26-09-2015

Revised: $18-11-2015$

Accepted: 19-11-2015

Corresponding Author: Ayodele Abraham Agboluaje School of Quantitative

Sciences, College of Arts and

Sciences, Universiti Utara

Malaysia

Department of Mathematics and Computer Science, Faculty of Natural Sciences, Ibrahim Badamasi Babangida

University, Lapai, Nigeria

Email: ayo_dele1960@yahoo.com

\begin{abstract}
This study has been able to reveal that the Combine White Noise model outperforms the existing Generalized Autoregressive Conditional Heteroscedasticity (GARCH) and Moving Average (MA) models in modeling the errors, that exhibits conditional heteroscedasticity and leverage effect. MA process cannot model the data that reveals conditional heteroscedasticity and GARCH cannot model the leverage effect also. The standardized residuals of GARCH errors are decomposed into series of white noise, modeled to be Combine White Noise model (CWN). CWN model estimation yields best results with minimum information criteria and high log likelihood values. While the EGARCH model estimation yields better results of minimum information criteria and high log likelihood values when compare with MA model. CWN has the minimum forecast errors which are indications of best results when compare with the GARCH and MA models dynamic evaluation forecast errors. Every result of CWN outperforms the results of both GARCH and MA.
\end{abstract}

Keywords: Determinant Residual Covariance, Minimum Forecast Errors, Minimum Information Criteria, Leverage, Log Likelihood

\section{Introduction}

The study is to model the error term that produces better and accurate results when compared with the existing models. The most important requirement in model building procedure in time series is that, the series must be stationary. The probability properties of the model assumed do not vary with time but changing with a fixed constant level mean and with constant variance. When there is an increase or decrease in the mean as time varies or as the variance is not constant with time, the series needs transformation to make it stationary for further computation to have reliable results. The results of model estimation can be spurious if the series is not stationary. The finite moving average procedure is constantly stationary (Said and Dickey, 1984; Harvey, 1993; Miettinen et al., 2014; Jentsch and Rao, 2015).

Moving average is the white noise error from previous periods. Moving average process is invertible if the coefficient of the white noise error is less than one. The residuals of moving average or mixed autoregressive moving average procedure will have equivalent residuals of properly selected autoregressive procedure. Therefore, the results from autoregressive procedure can be used in computing the variance or covariance matrix for autoregressive integrated moving average procedure by taking into consideration the equivalent variance or covariance matrix for the unpolluted autoregressive procedure. Tests of fit and diagnose checks by employing residuals autocorrelation of autoregressive model is applicable to moving average model (Box and Pierce, 1970; Miettinen et al., 2014).

The proposition approach of theory of testing autocorrelation when the lagged dependent variables of the regressors of a regression equation are integrated (Godfrey, 1978). This test is asymptotically corresponding to the suitable Likelihood Ratio (LR) test. This testing for the null hypothesis of serially uncorrelated against the alternative that a steady first order Autoregressive $\{$ AR (1) $\}$ process produces the errors of the regression model. This test is commonly 
employed in empirical studies. It is evident that the moving average error model of order $\mathrm{n}\{\mathrm{MA}(\mathrm{n})\}$ is a better hypothesis when compare with the AR(1) scheme, because the null hypothesis is that the moving average error are independently and normally distributed with zero mean and constant variance (Godfrey, 1978). Yet, there is no discovery of fourth order autocorrelation with this test (Godfrey, 1978).

The behaviour of the error term in the stochastic time series depends on the data size and high data frequencies. The error term can be white noise or heteroscedasticity, this dependent on the type of data. When the data exhibit heteroscedastic errors the moving average, autoregressive, mixed autoregressive moving average processes cannot model the heteroscedastic errors because of the non constant variances in the error term (Harvey, 1993).

Engle (1982) proposes autoregressive conditional heteroscedasticity (ARCH) model because of time varying volatility, to capture the effect of non constant variances in the error term. The equations are not normally distributed as there are changes in stock market distribution and fat tail measuring effect and this effect is named ARCH. ARCH models are able to grip group errors and can withstand any changes made by economic forecaster. But ARCH cannot handle the abnormalities like crashes, mergers, news effect or threshold effects in the financial and economic sector. ARCH can only model limited lag length. Bollerslev (1986) comes up with generalized ARCH to capture the volatility persistent that is flexible to uplift the weaknesses of ARCH model.

Vivian and Wohar (2012; Ewing and Malik, 2013) investigate that there are excess kurtosis and volatility persistence in GARCH which determines the asymmetric/leverage effects that GARCH cannot handled properly.

Threshold GARCH and exponential GARCH capture the asymmetric effects of positive and negative shocks of the same dimension on conditional volatility in various ways (Nelson, 1991; Hentschel, 1995; McAleer, 2014; McAleer and Hafner, 2014; Kamaruzzaman and Isa, 2015; Al-Hagyan et al., 2015; Farnoosh et al., 2015). GARCH modeling the leverage effect is not possible because any restriction imposed will be positivity restriction which has no leverage effect, since the negative correlation between returns shocks and subsequent shocks to volatility is the leverage effect (McAleer, 2014; McAleer and Hafner, 2014).

With these models weaknesses, CWN model is more appropriate to tackle these weaknesses.

CWN is computed by decomposing EGARCH error standardized results of unequal variances into equal variances series. Several statistics approaches and tests on CWN developments are detailed in the methodology and empirical data analysis of how the new model CWN is carried out to deal with conditional heteroscedastic errors and leverage effect.

\section{Materials and Methodology}

The data is retrieved from the DataStream of Universiti Utara Malaysia library. The U.S. Real Gross Domestic Product (GDP) quarterly data from 1960 to 2014 is used to investigate the efficiency of CWN model estimation when compared with the existing models.

Consider the model:

$y_{t}=\varphi y_{t-1}+\varepsilon_{t}$

Permit the stochastic approach of a real-valued time to be $\varepsilon_{t}$ and the complete information through $t$ time is $I$. The GARCH model is:

$$
\begin{aligned}
& \varepsilon_{t} \mid \mathrm{I}_{t-1} \sim \mathrm{N}\left(0, h_{t}\right) \\
& h_{t}=\omega+\sum_{i=1}^{q} \alpha_{i} \varepsilon_{t-i}^{2}+\sum_{i=1}^{p} \beta_{i} h_{t-i}=\omega+A(L) \varepsilon_{t}^{2}+B(L) h_{t}
\end{aligned}
$$

The EGARCH specification is:

$\log h_{t}=\alpha+\beta\left|z_{t-1}\right|+\delta z_{t-1}+\gamma \log h_{t-1},|\gamma|<1$

where, $z_{t}=\varepsilon_{t} / \sqrt{h_{t}}$ is the standardized shocks, $z_{t} \sim$ iid $(0, \alpha) .|\not|<1$ is when there is stability. The impact is asymmetric if $\delta \neq 0$, although, there is existence of leverage if $\delta<0$ and $\delta<\beta<-\delta$. While both $\beta$ and $\delta$ must be positive which the variances of two stochastic processes are, then, modeling leverage effect is not possible (McAleer, 2014; McAleer and Hafner, 2014).

The unequal variances (heteroscedastic errors) behaviors in the process of estimation being exhibited by GARCH models can be simplified into Combine White Noise models. The standardized residuals of GARCH errors which are unequal variances are decomposed into equal variances (white noise) in series to deal with the heteroscedasticity and leverage effect. The regression model is employed to transform each equal variances series to model.

Moving average process is employed for the estimation of these white noise series which is called Combine White Noise:

$$
\begin{aligned}
& Y_{1}=\varepsilon_{1 t}+\theta_{11} \varepsilon_{1, t-1}+\theta_{12} \varepsilon_{1, t-2}+\ldots \theta_{j q} \varepsilon_{j, t-q} \\
& Y_{2}=\varepsilon_{2 t}+\Phi_{21} \varepsilon_{2, t-1}+\Phi_{22} \varepsilon_{2, t-2}+\ldots \Phi_{j q} \varepsilon_{j, t-q} \\
& \vdots \\
& \quad \cdot \\
& Y j=\varepsilon_{j t}+\varphi_{j 1} \varepsilon_{j, t-1}+\varphi_{j 2} \varepsilon_{j, t-2}+\ldots \varphi_{j q} \varepsilon_{j, t-q} \\
& Y_{j t}=\sum_{j=1}^{q} \theta_{j} \varepsilon_{j, t-q}+\sum_{j=1}^{q} \Phi_{j} \varepsilon_{j, t-q}+\ldots \sum_{j=1}^{q} \varphi_{j} \varepsilon_{j, t-q}
\end{aligned}
$$




$$
\begin{aligned}
& =A(L) \varepsilon_{t}+B(L) \varepsilon_{t}+\ldots \\
& =\varepsilon_{t}[A(L)+B(L)+\ldots] \\
& =Q \varepsilon_{t}=U_{t}
\end{aligned}
$$

It can be written as:

$$
Y_{t}=U_{t}\left(U_{t} \sim \mathrm{N}\left(0, \sigma_{c}^{2}\right)\right.
$$

where, $A(L)+B(L)+\ldots=Q$ which are the matrix polynomial, $U_{t}$ is the error term of combine white noise model and $\sigma_{c}^{2}$ is the combination of equal variances. The combine variance of the combine white noise is:

$\sigma_{c}^{2}=\sigma_{1}^{2}+\sigma_{2}^{2}+\ldots$

Considering the two variances in the best two models of the first best models produced by the Bayesian model averaging output. The combine variance follows:

$\sigma_{c}^{2}=\sigma_{1}^{2}+\sigma_{2}^{2}$

The variance of errors, $\sigma_{c}^{2}$ in the combine white noise can be written:

$$
\sigma_{c}^{2}=W^{2} \sigma_{1}^{2}+(1-W)^{2} \sigma_{2}^{2}+2 \rho W \sigma_{1}(1-W) \sigma_{2}
$$

where, the balanced weight specified for the model is $W$. The least of $\sigma_{c}^{2}$ appearing, when the equation is differentiated with respect to $W$ and equate to zero, obtaining:

$$
W=\frac{\sigma_{c}^{2}-\rho \sigma_{1} \sigma_{2}}{\sigma_{1}^{2}+\sigma_{2}^{2}-2 \rho \sigma_{1} \sigma_{2}}
$$

where, $\rho$ is the correlation; intra-class correlation coefficient is used for a reliable measurement.

\section{Results}

The time plot of the data shows an upward trend which is a behavior of non-stationary.

The data is transformed in returns series to observe the volatility clustering, long tail skewness and excess kurtosis which are the characteristics of heteroscedasticity. The graph exhibits irregular variances that indicate volatility.

The Table 1 reports that, there are left long tail skewness, excess kurtosis and Jarque-Bera test is significant that is an indication of non-normality. Also standard deviation is less than one.

Table 1 shows the ARCH LM tests for the effect of heteroscedasticity in the data series; F-Statistic and Obs*R-squared are not significant that is an indication of $\mathrm{ARCH}$ presence in the data.
The Table 2 shows that the AIC, BIC and HQ minimum information criteria with log-likelihood that are used to select the appropriate model between $\mathrm{ARCH}$ and GARCH models. EGARCH model is choosing because it has minimum values of AIC, BIC and HQ with high log-likelihood values.

Combine White Noise $(\mathrm{CWN})$ has the minimum information criteria with high log likelihood. The CWN model gives the best results with minimum information criteria and high log likelihood when compare with GARCH and MA models estimation. The estimation of GARCH model and Combine White Noise model with their forecasting values are in Table 4.

In GARCH modeling, the leverage is not possible because any restriction imposed will be positivity restriction which has no leverage effect (McAleer, 2014; McAleer and Hafner, 2014). No proposition has removed heteroscedasticity completely (White, 1980; Antoine and Laveragne, 2014; Uchôa et al., 2014).

To avoid the above challenges, the standardized residuals graph of the GARCH model (GARCH errors) with unequal variances and zero mean are decomposed into equal variances series (white noise series). There are some graphs of equal variances (white noise series) with mean zero being obtained from graph of GARCH errors. These white noise series are fit into regression model to make each a model.

The implementation of Bayesian model averaging produces two best models from the first best models (Asatryan and Feld, 2014). For confirmations, fitting linear regression with autoregressive errors; 220 is the number of observation, with zero mean and variance one (Higgins and Bera, 1992). Therefore, the best two models are the white noise models.

Table 3 indicates an independent samples test is conducted to test whether data set of the two white noise models have equal variances or not. The test revealed that the variability in the distribution of the two data sets is no significantly different value which is greater than the p-value 0.05. Hence, the two models have equal variances (Lim and Loh, 1996; Boos and Brownie, 2004; Bast et al., 2015).

Table 4 reveals that Combine White Noise (CWN) appears to be the most appropriate model for estimation and forecasting, when comparing the three models.

Table 1. Histogram-normality and ARCH tests

\begin{tabular}{lll}
\hline & Coefficient/value & Probability \\
\hline Normal test & & \\
Standard deviation & 0.840452 & \\
Skewness & -0.320441 & \\
Kurtosis & 4.515921 & 0.000004 \\
Jarque-Bera & 24.71731 & \\
ARCH tests & & 0.2427 \\
F-Statistic & 1.372665 & 0.24 \\
Obs*R-squared & 1.376645 & \\
\hline
\end{tabular}


Table 2. ARCH, GARCH, MA, Combine White Noise (CWN) models

\begin{tabular}{lllllllll}
\hline & $\alpha$ & $\beta$ & $\delta$ & $\gamma$ & AIC & BIC & HQ & LL \\
\hline ARCH & 0.37700 & 0.14103 & & & 2.30379 & 2.42799 & 2.35396 & -243.11 \\
& $(0.000)$ & $(0.000)$ & & & & & & \\
EGARCH & 0.32771 & 0.32056 & -0.0656 & 0.89149 & 2.26776 & 2.37644 & 2.35396 & -240.19 \\
& $(0.000)$ & $(0.016)$ & $(0.397)$ & $(0.000)$ & & & & \\
MA & & & & & 11.2244 & 11.2709 & 11.2432 & -1226.1 \\
CWN & & & & & -0.5235 & -0.4306 & & 63.32035 \\
\hline
\end{tabular}

Note: $\alpha$ is the coefficient of the mean equation, $\beta$ and $\delta$ are the coefficients of the variance equations, while $\gamma$ is the coefficient of the $\log$ of variance equation. In the parentheses is the Probability Value (PV)

Table 3. Levene's test for equal variances

\begin{tabular}{|c|c|c|c|c|c|c|c|c|c|}
\hline & \multicolumn{9}{|c|}{ Independent samples test } \\
\hline & \multirow{2}{*}{\multicolumn{3}{|c|}{$\begin{array}{l}\text { Levene's test for } \\
\text { equality of variances }\end{array}$}} & \multicolumn{3}{|c|}{$\mathrm{t}$-test for equality of means } & \multirow{3}{*}{$\begin{array}{l}\text { Std.Error } \\
\text { difference }\end{array}$} & \multirow{2}{*}{\multicolumn{2}{|c|}{$\begin{array}{l}95 \% \text { Confidence interval } \\
\text { of the difference }\end{array}$}} \\
\hline & & & & & & & & & \\
\hline & $\mathrm{F}$ & Sig. & $\mathrm{t}$ & $\mathrm{df}$ & (2-tailed) & difference & & Lower & Upper \\
\hline B Equal variances assumed & 1.414 & 0.235 & 2.159 & 438 & 0.031 & 0.05909 & 0.02737 & 0.0053 & 0.11288 \\
\hline Equal variances not assumed & & & 2.159 & 255.236 & 0.032 & 0.05909 & 0.02737 & 0.00519 & 0.11299 \\
\hline
\end{tabular}

Table 4. The summary of MA, GARCH and Combine White Noise (CWN) models estimation and forecasting evaluation

\begin{tabular}{llll}
\hline & CWN & GARCH & MA \\
\hline $\begin{array}{l}\text { Estimation residual diagnostic } \\
\text { Stability test (Lag structure) }\end{array}$ & Stable & Stable & Stable \\
$\begin{array}{l}\text { Correlogram (square) residual } \\
\text { Portmanteau tests }\end{array}$ & $\begin{array}{l}\text { No autocorrelation } \\
\text { Histogram-normality tests }\end{array}$ & $\begin{array}{l}\text { Stationary } \\
\text { No autocorrelation }\end{array}$ & MA (1) serial correlation \\
ARCH test & Not normal & Not Normal & Not normal \\
Dynamic forecast evaluation & & No ARCH effect & ARCH effect \\
RMSE & 0.482821 & & \\
MAE & 0.113995 & 627.8018 & 305.8413 \\
MAPE & 1.387052 & 439.1633 & 237.8166 \\
Residual diagnostic & & 2.980324 & 1.658161 \\
Correlogram (square) residual & Stationary & & Stationary \\
Histogram-normality tests & Not normal & Stationary & Not normal \\
Serial correlation LM tests & No serial correlation & No serial correlation & No serial correlation \\
Heteroscedasticity test & No ARCH effect & No ARCH effect & No ARCH effect \\
Stability diagnostic & & & Stable \\
Ramsey reset tests & Stable & Stable & \\
Determinant residual covariance & 0.001923 & &
\end{tabular}

\section{Discussion}

Chuffart (2015) argues that Logistic Smooth Transition GARCH and Markov-Switching GARCH models are employed to confirm that Bayesian Information Criteria (BIC) can lead to wrong specification. CWN model employs Akaike Information Criteria (AIC), Bayesian Information Criteria (BIC) and log likelihood for specification of the model.

Chang et al. (2015) develop ample conditions for strict stationary and ergodicity of three nonlinear models of Self-Exciting Threshold Autoregressive (SETAR)GARCH process, the multiple-regime logistic Transition Auto Regressive (STAR) model with GARCH errors and Exponential STAR-GARCH model. They consider the STAR-GARCH models estimation results to be vital in financial econometrics. The development of CWN model is from GARCH family errors. CWN is tested using different countries data set with outstanding performance when compare with family GARCH model (EGARCH) which (Mutunga et al., 2015) considered to be suitable.

McAleer (2014) describes the asymmetry and leverage to be indistinguishable, and that leverage is asymmetry. The challenge is that there are no statistical properties for the estimation of this leverage effect. The estimation is only possible through positivity restriction of the parameters which is not an estimate for that can model the leverage effect. CWN model estimates with available statistical properties of maximum likelihood estimation to obtain efficient estimation and proves better than the estimation of the existing models.

McAleer and Hafner (2014) introduce one line derivation of EGARCH to model the asymmetric leverage effect, but in this process, stationarity and invertibility conditions are not determined. This makes it 
impossible to model the leverage effect. CWN model stationarity and invertibility are met.

Therefore, from these discussions, CWN model is suitable for efficient estimation.

\section{Conclusion}

It has been observed that the GARCH models have not been able to model the leverage effect, because when the positivity restriction is imposed, it has no leverage effect. The coefficient of variance equation must be negative for the existence of leverage (McAleer, 2014; McAleer and Hafner, 2014).

The standardized residual GARCH errors are decomposed into Combine White Noise (CWN). CWN has proved to be more efficient and it takes care of GARCH weaknesses. The estimation of Combine White Noise model passes stability condition, stationary, serial correlation, the ARCH effect tests and it also passes the Levene's test of equal variances.

The results in Table 2 and 4 show that CWN model estimation yield best results with minimum information criteria and high log likelihood values. While the EGARCH model estimation yield better results of minimum information criteria and high log likelihood values when compare with MA model. CWN has the minimum forecast errors which are indications of best results when compare with the GARCH and MA models dynamic evaluation forecast errors (Ismail and Muda, 2006; Fildes et al., 2011; Lazim, 2013). The determinant of the residual of covariance matrix value indicates that CWN is efficient.

Based on the every result in the empirical analysis, $\mathrm{CWN}$ is the most appropriate model. For this reason, $\mathrm{CWN}$ is recommended for the modeling of data that exhibits conditional heteroscedasticity and leverage effect.

The contribution of this study to the scientific community is that the CWN gives good results that improve the weaknesses of the existing models. CWN forecast output is more reasonable for effective policy making.

\section{Acknowledgement}

The authors thank the Universiti Utara Malaysia for the financial support in carrying out this research. The authors thank the reviewers who have taken their time to perfect this article.

\section{Author's Contributions}

Ayodele Abraham Agboluaje: Analyzing, producing the results and writing the paper.

Suzilah bt Ismail: Supervising the contents and flow of the paper.

Yin Chee Yip: Offering ideas, encouragement, proof read text and equations.

\section{Ethics}

This manuscript is original; there will be no expectation of any ethical issues after the publication. The three authors have read and approved the manuscript.

\section{References}

Al-Hagyan, M., M. Misiran and Z. Omar, 2015. Content analysis of stochastic volatility model in discrete and continuous time setting. Res. J. Applied Sci. Eng. Technol., 10: 1185-1191.

Antoine, B. and P. Lavergne, 2014. Conditional moment models under semi-strong identification. J. Econometr., 182: 59-69.

DOI: $10.1016 /$ j.jeconom.2014.04.008

Asatryan, Z. and L.P. Feld, 2014. Revisiting the link between growth and federalism: a Bayesian model averaging approach. J. Comparative Econom., 43: 772-781. DOI: 10.1016/j.jce.2014.04.005

Bast, A., W. Wilcke, F. Graf, P. Lüscher and H. Gärtner, 2015. A simplified and rapid technique to determine an aggregate stability coefficient in coarse grained soils. CATENA, 127: 170-176.

DOI: $10.1016 /$ j.catena.2014.11.017

Bollerslev, T., 1986. Generalized autoregressive conditional heteroskedasticity. J. Econometr., 31: 307-327. DOI: 10.1016/0304-4076(86)90063-1

Boos, D.D. and C. Brownie, 2004. Comparing variances and other measures of dispersion. Statist. Sci., 19: 571-578.

Box, G.E.P. and D.A. Pierce, 1970. Distribution of residual autocorrelations in autoregressiveintegrated moving average time series models. J. Am. Statist. Associat., 65: 1509-1526.

Chang, C.L., Y. Li and M. McAleer, 2015. Volatility spillovers between energy and agricultural markets: A critical appraisal of theory and practice.

Chuffart, T., 2015. Selection criteria in regime switching conditional volatility models. Economics, 3: 289316. DOI: 10.3390/econometrics3020289

Engle, R.F., 1982. Autoregressive conditional heteroscedasticity with estimates of the variance of United Kingdom inflation. Econometrica, 50: 987-1007. DOI: $10.2307 / 1912773$

Ewing, B.T. and F. Malik, 2013. Volatility transmission between gold and oil futures under structural breaks. Int. Rev. Econom. Finance, 25: 113-121. DOI: 10.1016/j.iref.2012.06.008

Farnoosh, R., M. Ebrahimi and S. Dalirian, 2015. Testing homogeneity of mixture of skew-normal distributions via Markov chain Monte Carlo simulation. Res. J. Applied Sci. Eng. Technol., 10: 112-117.

Fildes, R., Y. Wei and S. Ismail, 2011. Evaluating the forecasting performance of econometric models of air passenger traffic flows using multiple error measures. Int. J. Forecast., 27: 902-922. DOI: $10.1016 /$ j.ijforecast.2009.06.002 
Godfrey, L.G., 1978. Testing against general autoregressive and moving average error models when the regressors include lagged dependent variables. Econometrica, 46: 1293-1301. DOI: $10.2307 / 1913829$

Harvey, A.C., 1993. Time Series Models. 2nd Edn., The MIT Press, Cambridge. Massachusetts. Harvester Wheatsheaf Publisher.

Hentschel, L., 1995. All in the family nesting symmetric and asymmetric GARCH models. J. Financial Econom., 39: 71-104. DOI: $10.1016 / 0304-405 X(94) 00821-\mathrm{H}$

Higgins, M.L. and A.K. Bera, 1992. A class of nonlinear ARCH models. Int. Economic Rev., 33: 137-158. DOI: $10.2307 / 2526988$

Ismail, S. and T.Z. Muda, 2006. Comparing forecasting effectiveness through air travel data. Proceedings of Knowledge Management International Conference and Exhibition, Jun. 6-8, Sintok, pp: 594-602.

Jentsch, C. and S.S. Rao, 2015. A test for second order stationarity of a multivariate time series. J. Econometr., 185: 124-161.

DOI: 10.1016/j.jeconom.2014.09.010

Kamaruzzaman, Z.A. and Z. Isa, 2015. Modelling stock market return via normal mixture distribution. Res. J. Applied Sci., 10: 324-333. DOI: $10.3923 /$ rjasci.2015.324.333

Lazim, M.A., 2013. Introductory Business Forecasting: A Practical Approach. 3rd Edn., Penerbit Press, University Technology Mara, ISBN-10: 9789833643103, pp: 311.

Lim, T.S. and W.Y. Loh, 1996. A comparison of tests of equality of variances. Comput. Statist. Data Analysis, 22: 287-301.

DOI: $10.1016 / 0167-9473(95) 00054-2$
McAleer, M., 2014. Asymmetry and leverage in conditional volatility models. Econometrics, 2: 145-150. DOI: 10.3390/econometrics2030145

McAleer, M. and C.M. Hafner, 2014. A one line derivation of EGARCH. Econometrics, 2: 92-97. DOI: 10.3390 /econometrics2020092

Miettinen, J., K. Nordhausen, H. Oja and S. Taskinen, 2014. Deflation-based separation of uncorrelated stationary time series. J. Multivariate Analysis, 123: 214-227. DOI: 10.1016/j.jmva.2013.09.009

Nelson, D.B., 1991. Conditional heteroskedasticity in asset returns: A new approach. Econometrica, 59: 347-370. DOI: $10.2307 / 2938260$

Said, S.E. and D.A. Dickey, 1984. Testing for unit roots in autoregressive-moving average models of unknown order. Biometrika, 71: 599-607. DOI: $10.2307 / 2336570$

Uchôa, C.F.A., F. Cribari-Neto and T.A. Menezes, 2014. Testing inference in heteroskedastic fixed effects models. Eur. J. Operat. Res., 235: 660-670. DOI: $10.1016 /$ j.ejor.2014.01.032

Vivian, A. and M.E. Wohar, 2012. Commodity volatility breaks. J. Int. Financial Markets, Institut. Money, 22: 395-422. DOI: 10.1016/j.intfin.2011.12.003

White, H., 1980. A heteroskedasticity-consistent covariance matrix estimator and a direct test for heteroskedasticity. Econometrica, 48: 817-838. DOI: $10.2307 / 1912934$

Mutunga, T.N., A.S. Islam and L.A. Orawo, 2015. Implementation of the estimating functions approach in asset returns volatility forecasting using first order asymmetric GARCH models. Open J. Stati. DOI: $10.4236 /$ ojs.2015.55047 